\title{
A DNA Nanodevice That Loads and Releases a Cargo with Hemoglobin-Like Allosteric Control and Cooperativity
}

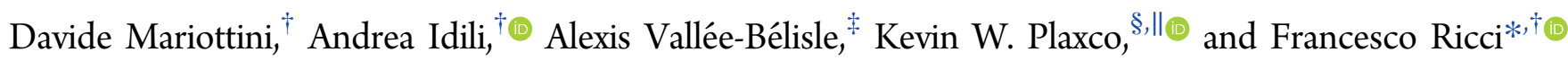 \\ ${ }^{\dagger}$ Department of Chemistry, University of Rome, Tor Vergata, Via della Ricerca Scientifica, 00133, Rome, Italy \\ ${ }^{\ddagger}$ Laboratory of Biosensors \& Nanomachines, Département de Chimie, Université de Montréal, C.P. 6128, Succursale Centre-Ville, \\ Montréal, Québec, Canada H3C 3J7 \\ ${ }^{\S}$ Department of Chemistry and Biochemistry, "Interdepartmental Program in Biomolecular Science and Engineering, University of \\ California Santa Barbara, Santa Barbara, California 93106, United States
}

Supporting Information

ABSTRACT: Here we report the rational design of a synthetic molecular nanodevice that is directly inspired from hemoglobin, a highly evolved protein whose oxygen-carrying activity is finely regulated by a sophisticated network of control mechanisms. Inspired by the impressive performance of hemoglobin we have designed and engineered in vitro a synthetic DNA-based nanodevice containing up to four interacting binding sites that, like hemoglobin, can load and release a cargo over narrow concentration ranges, and whose affinity can be finely controlled via both allosteric effectors and environmental cues like $\mathrm{pH}$ and temperature. As the first example of a synthetic DNA nanodevice that undergoes a complex network of nature-inspired control mechanisms, this represents an important step toward the use of similar nanodevices for diagnostic and drug-delivery applications.

KEYWORDS: DNA nanotechnology, molecular devices, DNA nanoswitches, DNA nanomachines, cooperativity

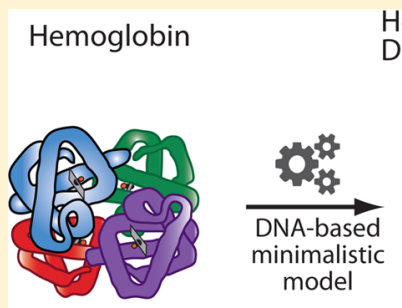

Hemoglobin-like DNA nanodevice
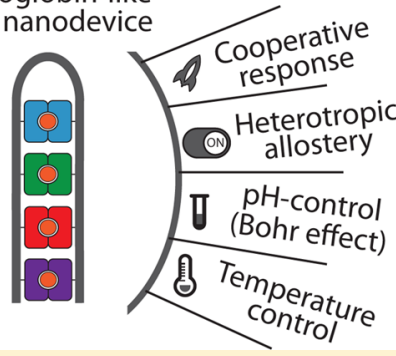
transport its cargo (i.e., oxygen) with great and finely tuned efficiency over even the narrow oxygen concentration gradients found in the body.

Here, inspired by the impressive performance of hemoglobin, we have engineered in vitro a single DNA nanodevice that features both hemoglobin's cooperative cargo binding (and release) ability and its efficient heterotropic allosteric regulation (by several physiological effectors). Specifically, we have developed a class of DNA-based nanodevices containing up to four interacting binding sites that load and release a molecular cargo over narrow concentration ranges, the midpoint placements of which are controlled via both allosteric effectors and by environmental cues (i.e., temperature and $\mathrm{pH}$ ).

Results and Discussion. Perhaps the most challenging property of hemoglobin to mimic is the homotropic allosteric control (i.e., cooperativity) with which it binds and releases oxygen. That is, the capacity of hemoglobin to load and release its cargo of oxygen over the narrow, $\sim 3$-fold difference in partial pressure that occurs between the lungs and the peripheral tissues. This effect is generated by the presence of four interacting oxygen-binding sites that work together such that binding to one improves binding to the others. This produces a high-order, nonlinear dependence of occupancy on oxygen concentration resulting in a steeper, sigmoidal binding curve. The physics of such cooperativity can be understood using the allosteric model first formulated by Monod et al., ${ }^{39}$ which describes the cooperative receptor as populating an equilibrium between two conformational states, one with low affinity (the "tense" state) and the other with high (the "relaxed" state) (in Figure $1 \mathrm{~A}$ is

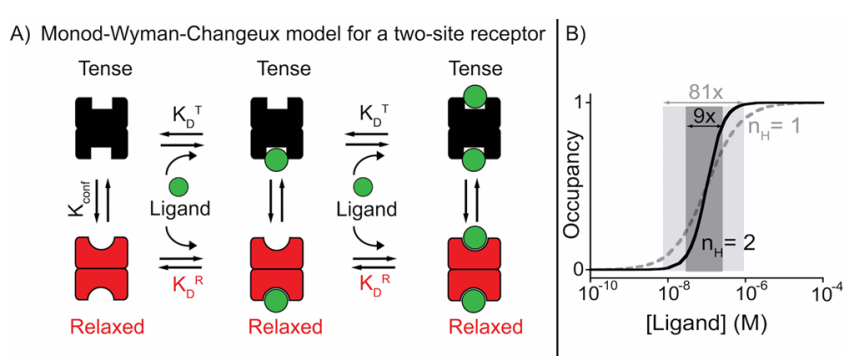

Figure 1. Two-site cooperative receptor. (A) The Monod, Wyman, and Changeux (MWC) model for a two-site cooperative receptor ${ }^{39}$ describes an equilibrium between two different conformations, one with low affinity for the ligand (called the "tense" state, $\mathrm{T}$ ) and another with higher affinity (called the "relaxed" state, R). Cooperativity arises when binding shifts this conformational equilibrium toward the higher affinity state with each successive binding event. This produces a steeper binding curve (ligand concentration versus occupancy) and a narrower dynamic range than that observed for simple, single-site binding. (B) Specifically, while a noncooperative receptor $\left(n_{\mathrm{H}}=1\right.$, gray curve; see eq 2) requires an 81 -fold change in ligand concentration to go from $10 \%$ occupancy to $90 \%$ occupancy, the same jump in occupancy for an ideal two-site cooperative receptor $\left(n_{\mathrm{H}}=2\right.$, black curve $)$ is achieved over only a 9-fold change in ligand concentration.

shown a minimalistic two-site example). Ligand binding to the low affinity state pushes this equilibrium toward the higher affinity state, increasing the likelihood of subsequent binding events and producing a steeper, higher-order dependence on ligand concentration. The resultant occupancy versus ligand concentration response can be conveniently fitted using the Hill equation: ${ }^{40}$

$$
\text { occupancy }=\frac{[\text { ligand }]^{n_{\mathrm{H}}}}{K_{1 / 2}^{n_{\mathrm{H}}}+[\text { ligand }]^{n_{\mathrm{H}}}}
$$

in which $K_{1 / 2}$ is the ligand concentration at which half of maximal occupancy is observed and $n_{\mathrm{H}}$ is the "Hill coefficient," which defines the degree of cooperativity. ${ }^{41}$ When $n_{\mathrm{H}}$ equals the number of binding sites, the receptor behaves as an ideally cooperative receptor exhibiting "all-or-none" ligand binding. The steeper dependence on concentration produced by this effect narrows the useful dynamic range of a receptor (here defined as the ratio between the free-ligand concentration that produces $10 \%$ occupancy, $C_{0.1}$, and that that produces $90 \%$ occupancy, $C_{0.9}$ ) with this range going as

$$
\text { dynamic range }=\frac{C_{0.9}}{C_{0.1}}=81^{1 / n_{\mathrm{H}}}
$$

For an ideally cooperative two-site receptor the useful dynamic range is thus narrowed to just 9-fold (as compared to the 81-fold of a noncooperative receptor), producing a much steeper binding curve (Figure 1B). To engineer hemoglobin-like allosteric cooperativity into a DNA-based nanodevice we initially considered a "minimalistic" model containing two binding sites recognizing a single-stranded DNA sequence as its ligand. As binding site we employed a clamp-like DNA sequence ${ }^{42}$ that binds its ligand to form a triple helix via simultaneous WatsonCrick and Hoogsteen base-pairing interactions (Figure 2A). To generate a cooperative nanodevice, we synthesized a construct consisting of two sequential copies of one-half of this binding site linked via a flexible 22-base, single-stranded loop to two sequential copies of its other half (Figure 2B). The entropic cost of closing this loop ensures that, in the absence of the target ligand, the construct is unfolded, with neither of its two binding sites being formed. The first binding event must thus pay the entropic cost of closing the loop, reducing its affinity. The second binding event, in contrast, occurs on a preformed binding site, improving its affinity as needed to generate a cooperative response. Our two-site construct exhibits cooperative binding. To see this we modified the two termini of the construct with a quencher/fluorophore pair, which reports ligand's binding via an easily measured decrease in fluorescence signal. Titrating this construct with a specific, 9-base target we obtain a binding curve with a Hill coefficient of $2.1 \pm 0.1$, which is within error of the 2.0 expected for an ideal two-site cooperative receptor (Figure 2B, right). This causes the useful dynamic range of the cooperative nanodevice to narrow to just $8.1( \pm 0.9)$-fold, rendering it much more sensitive to small changes in ligand concentration than would be a noncooperative receptor. In contrast, a control construct containing only a single recognition element (the second recognition element being altered so that it does not bind the same ligand) produces a Hill coefficient of $1.1 \pm 0.1$ and a dynamic range of $55 \pm 20$-fold, reflecting the expected, noncooperative binding (Figure 2C).

The relationship among cooperativity, the affinities of the high- and low-affinity states $\left(K_{\mathrm{D}}^{\mathrm{R}}\right.$ and $K_{\mathrm{D}}^{\mathrm{T}}$, respectively), and the equilibrium constant for the conformational shift between the two $\left(K_{\text {conf }}\right)$ is given in the MWC model by

$$
=\frac{\frac{[L]}{K_{\mathrm{D}}^{\mathrm{R}}}\left(1+\frac{[L]}{K_{\mathrm{D}}^{\mathrm{R}}}\right)^{n-1}+K_{\text {conf }} \times \frac{K_{\mathrm{D}}^{\mathrm{R}}}{K_{\mathrm{D}}^{\mathrm{T}}} \frac{[L]}{K_{\mathrm{D}}^{\mathrm{T}}}\left(1+\frac{K_{\mathrm{D}}^{\mathrm{R}}}{K_{\mathrm{D}}^{\mathrm{T}}} \frac{[L]}{K_{\mathrm{D}}^{\mathrm{R}}}\right)^{n-1}}{\left(1+\frac{[L]}{K_{\mathrm{D}}^{\mathrm{R}}}\right)^{n}+K_{\operatorname{conf}}\left(1+\frac{K_{\mathrm{D}}^{\mathrm{R}}}{K_{\mathrm{D}}^{\mathrm{T}}} \frac{[L]}{K_{\mathrm{D}}^{\mathrm{R}}}\right)^{n}}
$$

where $[\mathrm{L}]$ is the ligand concentration and $n$ is the number of binding sites. To determine $K_{\mathrm{D}}{ }^{\mathrm{R}}, K_{\mathrm{D}}{ }^{\mathrm{T}}$, and $K_{\text {conf }}$ for our system, we designed several control nanodevices (Figure 3 ). The first 
A) Clamp-like triplex forming mechanism

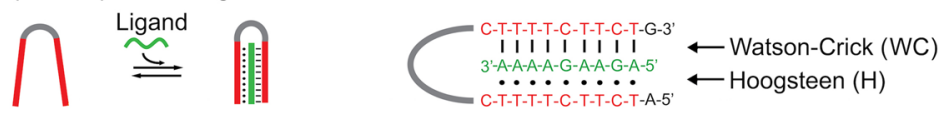

B) Cooperative DNA-Nanodevice

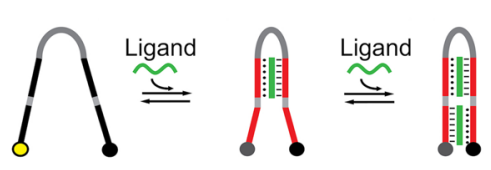

C) Non-cooperative DNA-Nanodevice
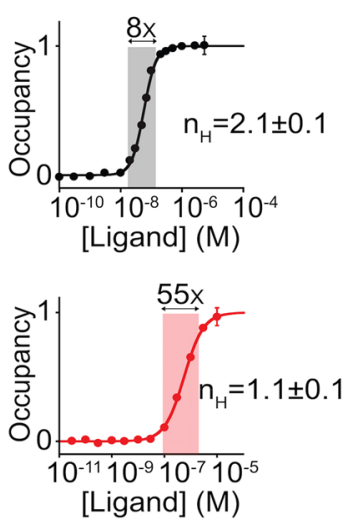

Figure 2. Cooperative DNA-nanodevice. (A) To rationally design our cooperative DNA nanodevice we employed as the recognition element a triplex forming DNA sequence. This behaves like a "clamp" that binds a specific 9-base DNA ligand via the formation of both Watson-Crick and Hoogsteen base-pair interactions. (B) The cooperative DNA nanodevice is obtained by joining together two sequential copies of one-half of such recognition element linked via a flexible 22-base, single-stranded loop (gray portion) to two sequential copies of its other half. Binding of the ligand to the first receptor decreases the entropic cost associated with the binding to the second receptor (and thus improves its affinity for the ligand). As a result, this nanodevice shows a Hill-type cooperative response. In contrast, a similar nanodevice containing only a single binding site does not exhibit cooperativity (panel C, $n_{\mathrm{H}}=1.1 \pm 0.1$ ). The binding curves presented here and in the following figures were obtained using a DNA nanodevice (either cooperative or control) at $3 \mathrm{nM}$ concentration and adding increasing concentrations of ligand DNA at $\mathrm{pH} 7.5$ and $35^{\circ} \mathrm{C}$ unless otherwise noted. Here and in the following figures, the experimental values represent averages of three separate measurements, and the error bars reflect the standard deviations.

A) Control DNA nanodevice (Tense state)

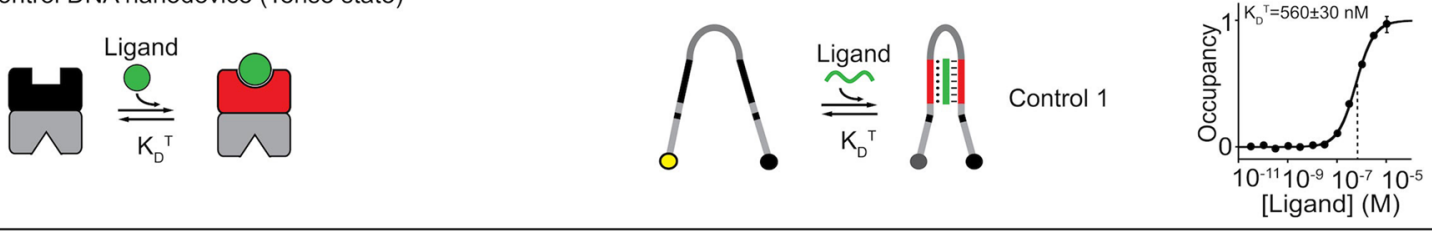

B) Control DNA nanodevice (Relaxed state)
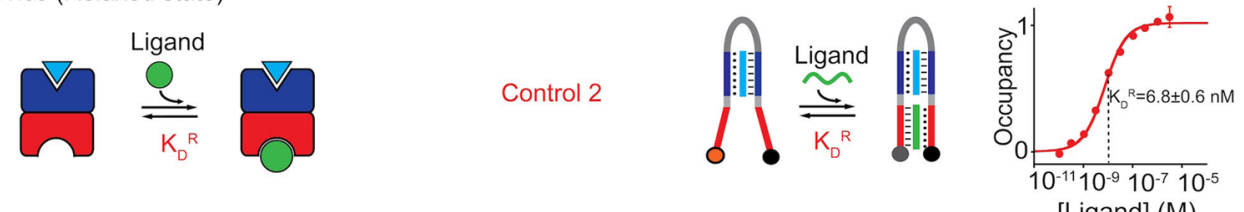

C) Cooperative DNA nanodevice
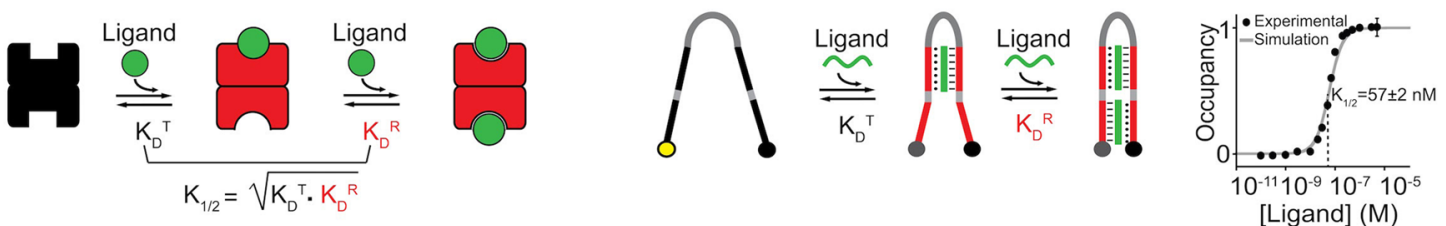

Figure 3. Modeling the cooperative DNA nanodevices. To dissect the cooperative behavior of our DNA nanodevice using the MWC model, we have characterized a number of "control" constructs. (A) The first contains only a single binding site (the "upper" binding site) and thus can be used to estimate the affinity of lower-affinity state of the cooperative nanodevice $\left(K_{\mathrm{D}}^{\mathrm{T}}\right)$. (B) We used a nanodevice containing two different binding sites-with the lower binding site recognizing the same ligand as the cooperative nanodevice-to estimate the affinity of higher-affinity state of the cooperative nanodevice $\left(K_{\mathrm{D}}^{\mathrm{R}}\right)$ by making the measurement in the presence of a high concentration of the upper binding site's ligand. (C) Plugging these estimated affinities into the MWC model recapitulates the cooperative behavior (solid curves) of our nanodevice without the use of any fitted parameters.

control employed the loop and first recognition element of the cooperative nanodevice but replaced the second recognition element with sequences that do not bind the ligand (Figure 3A). This provides a means of determining the affinity of the loweraffinity "tense" state $\left(K_{\mathrm{D}}^{\mathrm{T}}=560 \pm 30 \mathrm{nM}\right)$. The second control nanodevice contains two recognition elements (Figure $3 \mathrm{~B}$ ), with one (red) recognizing the same ligand sequence of our cooperative nanodevice and the other recognizing a second, different ligand. By adding a high concentration of such alternative ligand (blue strand) we forced the system into its high-affinity "relaxed" state, providing a means of measuring its affinity. Doing so we found that the affinity of this state, $K_{D}{ }^{R}$, is $6.8 \pm 0.6 \mathrm{nM}$. 


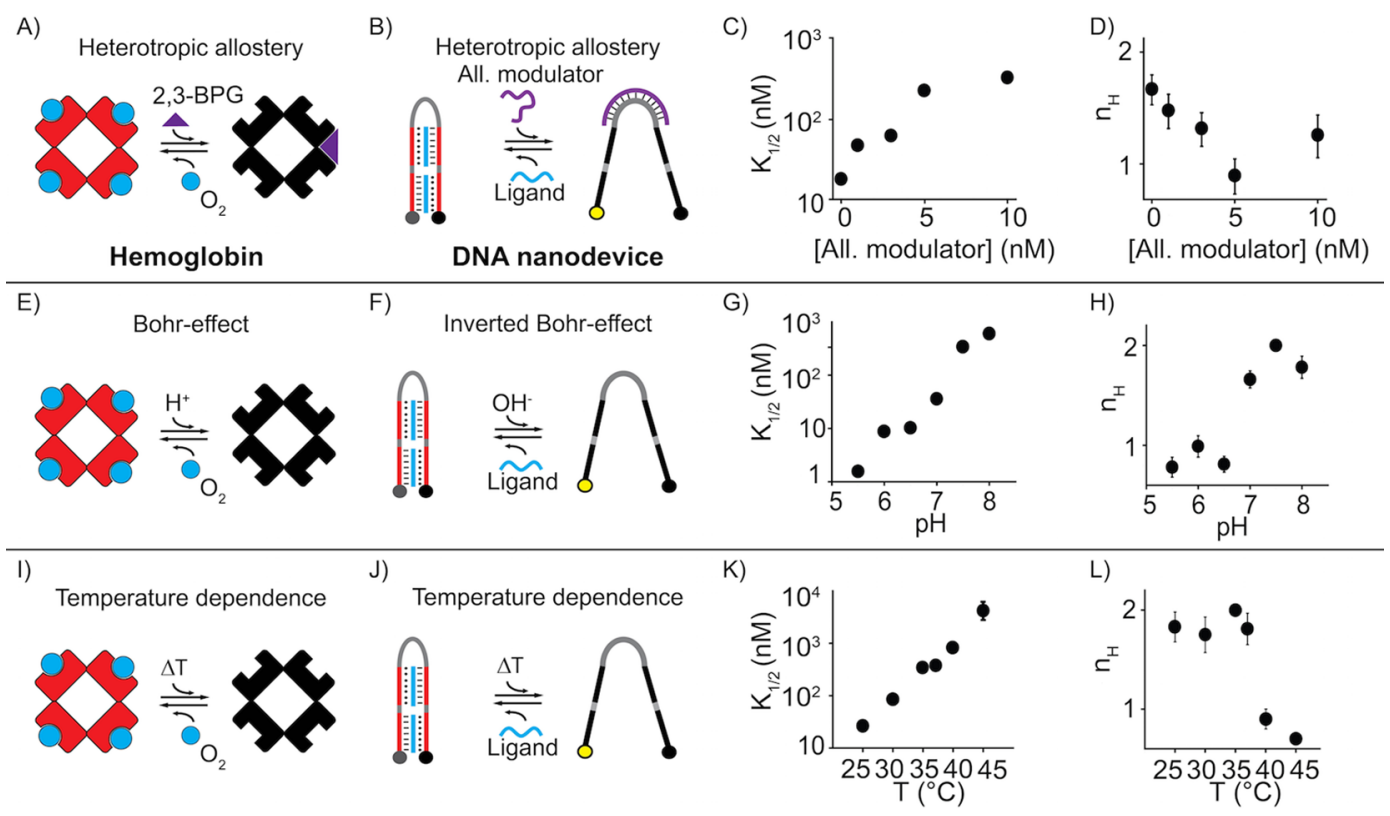

Figure 4. Allosteric control of the DNA nanodevice. (A) The binding of the nanodevice can be controlled via the introduction of allosteric effectors in a manner similar to that seen for hemoglobin. (B) For example, when bound to the nanodevice a DNA sequence complementary to the loop increases the entropic cost associate with ligand binding. (C) As a result, the effector shifts the equilibrium of the nanodevice toward the lower affinity state, pushing the midpoint of the ligand binding curve to higher concentrations. (D) Binding of the allosteric modulator also affects the cooperativity of the nanodevice. (E) The Bohr effect describes the $\mathrm{pH}$ dependency of hemoglobin, which exhibits poorer oxygen binding affinity at lower $\mathrm{pH}$. ( $\mathrm{F}$ ) The binding of our DNA nanodevice is similarly under $\mathrm{pH}$ control, albeit in this case we observe an "inverted" Bohr effect as affinity increases at lower $\mathrm{pH}$. (G) By modulating the $\mathrm{pH}$ of the solution, we can thus finely tune both the affinity of the nanodevice and (H) its cooperativity. (I) Finally, like the binding efficiency of hemoglobin for oxygen is regulated by temperature, also the affinity $(\mathrm{J}-\mathrm{K})$ and cooperativity $(\mathrm{L})$ of the nanodevice is likewise temperature-dependent.

Finally, we estimated $K_{\text {conf }}$ the conformational equilibrium constant between the two states, from the ratio of the affinity constants of the cooperative, two-site nanodevice described above to that of a nanodevice where the 20-base loop is substituted with a 5-base loop (Figure S1); this short loop minimizes the entropic cost associated with the closure of the nanodevice upon the first binding event, enhancing the population of the high affinity state. From the affinity constant of this control system, $5.1 \pm 1.1 \mathrm{nM}$, we estimated that $K_{\text {conf }}=67 \pm 17$. Together these parameters describe our cooperative nanodevice. Specifically, the $K_{1 / 2}, 58 \pm 20 \mathrm{nM}$, and the Hill coefficient, $1.6 \pm$ 0.2 , predicted by placing these values into eq 3 (the MWC model) are in reasonably good agreement with the values, $57 \pm$ $2 \mathrm{nM}$ and $2.1 \pm 0.1$, respectively, experimentally observed for our cooperative nanodevice (Figure 3C).

To better mimic the hemoglobin-like cooperativity, we have also designed nanodevices containing as many as four binding sites. The maximum Hill coefficient these have achieved, however, is just $2.4 \pm 0.2$ (for a 3 binding site construct; Figure S2). The fact that our nanomachine does not reach the ideal maximum Hill coefficient (i.e., corresponding to the total number of binding sites) is not too surprising. Nature, in fact, also struggles with multisite receptors. For example, the Hill coefficient of hemoglobin, with only 3.2 , is far from the optimal value predicted with four binding sites. ${ }^{43}$ We suspect that in the case of our nanomachine the deviation from the ideal behavior might be due to nonspecific interactions among the binding elements in the construct which would inevitably disrupt the delicate balance required to achieve optimal cooperativity.

In addition to cooperativity (homotropic allostery, in which the binding of oxygen modulates further oxygen binding), hemoglobin also exhibits heterotropic allostery, in which its oxygen affinity is modulated via the binding of other molecular effectors at sites distal from the oxygen binding sites. The effector 2,3-bisphosphoglycerate (2,3-BPG), for example, binds mammalian hemoglobin stabilizing its low affinity state and thus shifting the oxygen-binding curve to higher concentrations (Figure 4A). We have produced a similar effect on a variant of our DNA nanodevice by designing a DNA strand complementary to the unstructured loop (which is, of course, distal from the ligand binding site; Figure 4B). The binding of this effector renders the formation of the binding competent conformation more difficult, thus affecting its affinity for the ligand (Figure 4C). Specifically, the $K_{1 / 2}$ of the nanodevice shifts from $17 \pm 1 \mathrm{nM}$ to $335 \pm 40 \mathrm{nM}$ as the concentration of the effector rises from 0 to $10 \mathrm{nM}$ (Figure 4C and Figure S3). Because effector binding alters the device's conformational equilibrium constant $\left(K_{\text {conf }}\right)$, the effector also alters the cooperativity of the nanodevice (see eq 3 above), shifting $n_{\mathrm{H}}$ from $1.6 \pm 0.1$ to $1.2 \pm 0.1$ over the same range of effector concentrations (Figure 4D).

In addition to being modulated by oxygen and BPG, hemoglobin's activity is also controlled by $\mathrm{pH}$ via the Bohr effect, first described by Bohr et al. in $1904 .{ }^{44}$ Specifically, hemoglobin's oxygen affinity is inversely related to acidity (Figure 4E). Because triplex formation requires the protonation of cytosines $\left(\mathrm{p} K_{\mathrm{a}}=\right.$ $6.5),{ }^{42,45-48}$ the affinity of our DNA cooperative nanodevices is likewise $\mathrm{pH}$-dependent, albeit in an opposite sense to hemoglobin. That is, our nanodevice exhibits an "inverted" Bohr effect in which its affinity is directly, rather than inversely, related to acidity (Figure 4F). For example, shifting the $\mathrm{pH}$ from 8.0 to 5.5 reduces $K_{1 / 2}$ from $600 \pm 30 \mathrm{nM}$ to $1.6 \pm 0.3 \mathrm{nM}$ (Figure $4 \mathrm{G}$ and Figure S4). Finally, as it occurs with some hemoglobins (such as those found in fish ${ }^{49}$ and cats ${ }^{50}$ ), the cooperativity of our nanodevice is also $\mathrm{pH}$-dependent, with maximum cooperativity 
occurring under alkaline conditions (Figure $4 \mathrm{H}$ ). This is likely due to the fact that, under acidic conditions, even the lower affinity state binds the ligand with a rather high affinity, reducing cooperativity.

Hemoglobin's affinity for oxygen is also modulated by temperature, ${ }^{43}$ with its oxygen affinity decreasing as the temperature rises (Figure 4I). This improves the efficiency of oxygen release in metabolizing tissues, where the temperature is usually elevated. Our nanodevice shows similar temperature dependence, with both its ligand affinity and cooperativity falling as the temperature rises (Figure $4 \mathrm{~J}-\mathrm{L}$ and Figure S5), presumably due to the increase of the entropic contribution cost of closing the loop during the first binding event.

Conclusions. Inspired by one of the most highly optimized biomolecular machines found in living systems, hemoglobin, we have rationally designed a synthetic, DNA-based nanodevice that simultaneously exhibits both homotropic cooperativity and heterotropic allosteric control. Specifically, we have developed a class of DNA-based nanodevices containing up to four allosterically interacting binding sites that can load and release a specific DNA strand cargo and whose binding efficiency can be finely controlled via both allosteric molecular effectors and environmental cues. For example, the high Hill coefficients of our DNA-based nanodevice allow the loading and release of its ligand over narrow concentration ranges, as is true for the oxygen carrying capacity of hemoglobin. Likewise, we can finely control the binding affinity of the DNA-based nanodevice for its ligand via the introduction of specific allosteric effectors. Finally, like hemoglobin, the cooperative response and binding efficiency of our DNA-based nanodevice is controlled by both temperature and $\mathrm{pH}$.

Hemoglobin relies on a highly complex multimeric structure comprising in total more than 570 amino acids (and four ironcontaining heme prostetic groups) to generate homotropic and heterotropic allostery and is the result of millions of years of evolutionary optimization. Here, by taking advantage of the predictability of DNA base-pairing interactions, we have engineered a much smaller, minimalistic model that achieves many of the same features. Despite the fact that the performance of our DNA-based nanodevice is still not comparable to that of hemoglobin, the results we have achieved represent a promising indication that DNA nanotechnology might be a preferred route to recapitulate in synthetic nanodevices regulatory mechanisms and strategies that nature has evolved for controlling and enhancing biomolecular function. Because DNA sequences can be selected or modified to specifically bind targets other than DNA (small molecules, proteins, antibodies, etc.), the strategy we have demonstrated here could be generalized to design highly controlled DNA-based nanodevices that can load and release a wide range of molecular cargoes.

Materials and Methods. Chemicals, Oligonucleotides, and DNA-Based Nanodevice. All reagent-grade chemicals, including Tris $\mathrm{HCl}$ and $\mathrm{MgCl}_{2}$ (all from Sigma-Aldrich, St. Louis, Missouri), were used as received. HPLC purified oligonucleotides were purchased from IBA (Gottingen, Germany) or Biosearch Technologies (Risskov, Denmark). The DNA strand for the nanodevices were modified with Alexa Fluor 488 and BHQ-1 (black hole quencher 1). The sequences and modification schemes are as follows.

-Cooperative DNA-nanodevice (two binding sites): $5^{\prime}$-(Alexa488) ACT TTT CTT CTT CTT CTT TTC AGT TAT TAT TAG TTA TTA TTC TTT TCT TCT TCT TCT TTT CG (BHQ1)-3'.
-Cooperative DNA-nanodevice (three binding sites): 5'-(Alexa488) ATC TTC TTT TCC TTT TCT TCT ТCT TCT TTT CAG TTA TTA TTA GTT ATT ATT CTT TTC TTC TTC TTC TTT TCC TTT TCT TCT G (BHQ1)-3'.

-Cooperative DNA-nanodevice (four binding sites): 5'-(Alexa488) ACT TTT CTT CTT CTT CTT TTC CTT TTC TTC TTC TTC TTT TCA GTT ATT ATT AGT TAT TAT TCT TTT CTT СTT CTT CTT TTC СTT TTC TTC TTC TTC TTT TCG (BHQ1)-3'.

-DNA nanodevice (control also used to measure $K_{D}{ }^{T}$ ): 5'-(Alexa488) AGT GGA GGA GTT CTT CTT TTC AGT TAT TAT TAG TTA TTA TTC TTT TCT TCT CTT TTC TTC TG (BHQ1)-3'.

-DNA nanodevice (used to measure $\left.K_{D}{ }^{R}\right): 5^{\prime}$-(Alexa488) ACT TTT CTT CTC CTT CCC TTT AGT TAT TAT TAG TTA TTA TTT TTC CCT TCC TCT TCT TTT CG (BHQ1)-3'. -DNA ligand 9-base: 5'-AGA AGA AAA-3'.

-DNA ligand 10-base (used to measure $K_{D}{ }^{R}$ ): $5^{\prime}$-GGA AGG GAA A-3'.

-Cooperative DNA-nanodevice (allosteric effect): 5'-(Alexa488) ACT TTT CTT СTT СTT CTT TTC AAG TTA TTA TTC TCT TTT CTT CTT CTT CTT TTC G (BHQ1)-3'.

-DNA allosteric effector 15 base: 5'-GAG AAT AAT AAC TTG-3'.

-DNA nanodevice (loop 5 base): 5'-(Alexa488) ACT TTT CTT CTT CTT CTT TTC AGT TAC TTT TCT TCT TCT TCT TTT CG (BHQ1)-3'.

Fluorescent Experiments. Fluorescent experiments were conducted in $10 \mathrm{mM}$ Tris, $2 \mathrm{mM} \mathrm{MgCl}_{2}$ at $25{ }^{\circ} \mathrm{C}$ (unless otherwise noted). Equilibrium fluorescence measurements were obtained using a Cary Eclipse fluorimeter with excitation at $490( \pm 5) \mathrm{nm}$ (for DNA strands labeled with Alexa Fluor 488) and acquisition at $517( \pm 5) \mathrm{nm}$. Binding curves were obtained in a $800 \mu \mathrm{L}$ solution containing 3 or $10 \mathrm{nM}$ of DNA-nanodevices and sequentially adding increasing concentrations of the DNA ligand. For each concentration the fluorescence signal was recorded every $20 \mathrm{~min}$ until it reached equilibrium. The signals obtained have been normalized on a $0-1$ scale to allow for more ready interpretation of the results. More specifically, the relative occupancy (defined as the fraction of nanomachine bound to the ligand) was plotted against the ligand concentration. To obtain the relative occupancy, we considered the maximum signal of the nanomachine as the signal of the unbound nanomachine (occupancy $=0$ ), while the minimum signal was considered as the signal of the completely bound nanomachine at saturating concentration of ligand (occupancy $=1$ ).

To determine the Hill coefficient, we measured fluorescence of five replicate points per titration. We used KaleidaGraph plotting software to fit the traces to the Hill equation. Error bars represent $95 \%$ confidence intervals based on standard errors derived from the fits.

The observed fluorescence, $F_{[\text {ligand }]}$, was fitted using the following four-parameter logistic equation:

$$
F_{[\text {ligand }]}=F_{\min }+\frac{\left(F_{\max }[\text { ligand }]^{n_{\mathrm{H}}}\right)}{\left[\left(K_{1 / 2}\right)^{n_{\mathrm{H}}}+[\text { ligand }]^{n_{\mathrm{H}}}\right]}
$$

where $F_{\min }$ and $F_{\max }$ are the minimum and maximum fluorescence values, $K_{1 / 2}$ is the equilibrium ligand concentration at halfmaximum signal, $n_{\mathrm{H}}$ is the Hill coefficient, and [ligand] is the concentration of the ligand oligonucleotide added. 


\section{ASSOCIATED CONTENT}

\section{S Supporting Information}

The Supporting Information is available free of charge on the ACS Publications website at DOI: 10.1021/acs.nanolett.7b00814.

Supporting figures (PDF)

\section{AUTHOR INFORMATION}

\section{Corresponding Author}

*E-mail: francesco.ricci@uniroma2.it.

ORCID $\odot$

Andrea Idili: 0000-0002-6004-270X

Kevin W. Plaxco: 0000-0003-4772-8771

Francesco Ricci: 0000-0003-4941-8646

Author Contributions

D.M. and A.I. contributed equally.

Notes

The authors declare no competing financial interest.

\section{ACKNOWLEDGMENTS}

This work was supported by Associazione Italiana per la Ricerca sul Cancro, AIRC (project no. 14420) (FR), by the European Research Council, ERC (project no. 336493) (FR), by the Int. Research Staff Exchange Scheme (IRSES) (FR), and by the National Sciences and Engineering Research Council of Canada through Grant No. 436381-2013 (NSERC) (A.V.-B.).

\section{REFERENCES}

(1) Browne, W. R.; Feringa, B. L. Nat. Nanotechnol. 2006, 1, 25-35.

(2) Abendroth, J. M.; Bushuyev, O. S.; Weiss, P. S.; Barrett, C. J. ACS Nano 2015, 9, 7746-7768.

(3) Ragazzon, G.; Baroncini, M.; Silvi, S.; Venturi, M.; Credi, A. Nat. Nanotechnol. 2014, 10, 70-75.

(4) Koumura, N.; Zijlstra, R. W. J.; van Delden, R. A.; Harada, N.; Feringa, B. L. Nature 1999, 401, 152-155.

(5) Balzani, V.; Credi, A.; Raymo, F. M.; Stoddart, J. F. Angew. Chem., Int. Ed. 2000, 39, 3348-3391.

(6) Hernández, J. V.; Kay, E. R.; Leigh, D. A. Science 2004, 306, 15321537.

(7) Ma, X.; Hortelão, A. C.; Patiño, T.; Sánchez, S. ACS Nano 2016, 10, 9111-9122.

(8) Gao, W.; Wang, J. Nanoscale 2014, 6, 10486-10494.

(9) Mattia, E.; Otto, S. Nat. Nanotechnol. 2015, 10, 111-119.

(10) Zhao, Y. X.; Shaw, A.; Zeng, X.; Benson, E.; Nyström, A. M.; Högberg, B. ACS Nano 2012, 6, 8684-8691.

(11) Krishnan, Y.; Simmel, F. C. Angew. Chem., Int. Ed. 2011, 50, 3124-3156.

(12) Yurke, B.; Turberfield, A. J.; Mills, A. P., Jr.; Simmel, F. C.; Neumann, J. L. Nature 2000, 406, 605-608.

(13) Wickham, S. F. J.; Bath, J.; Katsuda, Y.; Endo, M.; Hidaka, K.; Sugiyama, H.; Turberfield, A. J. Nat. Nanotechnol. 2012, 7, 169-173.

(14) Jester, S. S.; Famulok, M. Acc. Chem. Res. 2014, 47, 1700-1709.

(15) Liedl, T.; Sobey, T.; Simmel, F. C. Nano Today 2007, 2, 36-41.

(16) Modi, S.; Nizak, C.; Surana, S.; Halder, S.; Krishnan, Y. Nat. Nanotechnol. 2013, 8, 459-467.

(17) Surana, S.; Bhat, J. M.; Koushika, S. P.; Krishnan, Y. Nat. Commun. 2011, 2, 340.

(18) Bath, J.; Turberfield, A. J. Nat. Nanotechnol. 2007, 2, 275-284.

(19) Andersen, E. S.; Dong, M.; Nielsen, M. M.; Jahn, K.; Subramani, R.; Mamdouh, W.; Golas, M. M.; Sander, B.; Stark, H.; Oliveira, C. L. P.; Pedersen, J. S.; Birkedal, V.; Besenbacher, F.; Gothelf, K. V.; Kjems, J. Nature 2009, 459, 73-76.

(20) Douglas, S. M.; Dietz, H.; Liedl, T.; Högberg, B.; Graf, F.; Shih, W. M. Nature 2009, 459, 414-418.
(21) Kuzyk, A.; Yang, Y.; Duan, X.; Stoll, S.; Govorov, A. O.; Sugiyama, H.; Endo, M.; Liu, N. Nat. Commun. 2016, 7, 10591.

(22) Meng, W.; Muscat, R. A.; McKee, M. L.; Milnes, P. J.; El-Sagheer, A. H.; Bath, J.; Davis, B. G.; Brown, T.; O’Reilly, R. K.; Turberfield, A. J. Nat. Chem. 2016, 8, 542-548.

(23) Krishnan, S.; Ziegler, D.; Arnaut, V.; Martin, T. G.; Kapsner, K.; Henneberg, K.; Bausch, A. R.; Dietz, H.; Simmel, F. C. Nat. Commun. 2016, 7, 12787.

(24) Chen, Y.-J.; Groves, B.; Muscat, R. A.; Seelig, G. Nat. Nanotechnol. 2015, 10, 748-760.

(25) Surana, S.; Shenoy, A. R.; Krishnan, Y. Nat. Nanotechnol. 2015, 10, $741-747$.

(26) Jones, M. R.; Seeman, N. R; Mirkin, C. Science 2015, 347, 1260901.

(27) Pinheiro, A. V.; Han, D.; Shih, W. M.; Yan, H. Nat. Nanotechnol. 2011, 6, 763-772.

(28) Simon, A. J.; Vallée-Bélisle, A.; Ricci, F.; Plaxco, K. W. Proc. Natl. Acad. Sci. U. S. A. 2014, 111, 15048-15053.

(29) Ricci, F.; Vallée-Bélisle, A.; Plaxco, K. W. PLoS Comput. Biol. 2011, 7, e1002171.

(30) Ricci, F.; Vallée-Bélisle, A.; Porchetta, A.; Plaxco, K. W. J. Am. Chem. Soc. 2012, 134, 15177-15180.

(31) Ricci, F.; Vallée-Bélisle, A.; Simon, A. J.; Porchetta, A.; Plaxco, K. W. Acc. Chem. Res. 2016, 49, 1884-1892.

(32) Adair, G. S. J. Biol. Chem. 1925, 63, 529-545.

(33) Changeux, J.-P.; Edelstein, S. J. Neuron 1998, 21, 959-980.

(34) Changeux, J.-P. Protein Sci. 2011, 20, 1119-1124.

(35) Eaton, W. A.; Henry, E. R.; Hofrichter, J.; Mozzarelli, A. Nat. Struct. Biol. 1999, 6, 351-358.

(36) Ferrell, J. E., Jr. Trends Biochem. Sci. 1996, 21, 460-466.

(37) Koshland, D. E., Jr.; Nemethy, G.; Filmer, D. Biochemistry 1966, 5, 365-385.

(38) Yuan, Y.; Tam, M. F.; Simplaceanu, V.; Ho, C. Chem. Rev. 2015, $115,1702-1724$.

(39) Monod, J.; Wyman, J.; Changeux, J.-P. J. Mol. Biol. 1965, 12, 88118.

(40) Hill, A. V. J. Physiol. 1910, 40, 190-224.

(41) Ferrell, J. E., Jr. J. Biol. 2009, 8, 53-56.

(42) Idili, A.; Plaxco, K. W.; Vallée-Bélisle, A.; Ricci, F. ACS Nano 2013, 7, 10863-10869.

(43) Reeves, R. B. Respir. Physiol. 1980, 42, 317-328.

(44) Bohr, C.; Hasselbalch, K.; Krogh, A. Skand. Arch. Physiol. 1904, 16, 402-412.

(45) Ohmichi, T.; Kawamoto, Y.; Wu, P.; Miyoshi, D.; Karimata, H.; Sugimoto, N. Biochemistry 2005, 44, 7125-7130.

(46) Leitner, D.; Schröder, W.; Weisz, K. Biochemistry 2000, 39, 58865892.

(47) Sugimoto, N.; Wu, P.; Hara, H.; Kawamoto, Y. Biochemistry 2001, 40, 9396-9405.

(48) Idili, A.; Vallée-Bélisle, A.; Ricci, F. J. Am. Chem. Soc. 2014, 136, 5836-5839.

(49) Tan, A. L.; De Young, A.; Noble, R. W. J. Biol. Chem. 1972, 25, 2493-2498.

(50) Hamilton, M. N.; Edelstein, S. J. Science 1972, 178, 1104-1106. 